\title{
Sinc Collocation Method for Finding Numerical Solution of Integrodifferential Model Arisen in Continuous Mixed Strategy
}

\author{
F. Hosseini Shekarabi \\ Department of Mathematics, Shahid Rajaee Teacher Training University, Lavizan, Tehran, Iran \\ Correspondence should be addressed to F. Hosseini Shekarabi; f_hosseini@srttu.edu
}

Received 22 February 2014; Revised 9 August 2014; Accepted 21 August 2014; Published 17 September 2014

Academic Editor: Fu-Yun Zhao

Copyright ( 2014 F. Hosseini Shekarabi. This is an open access article distributed under the Creative Commons Attribution License, which permits unrestricted use, distribution, and reproduction in any medium, provided the original work is properly cited.

One of the new techniques is used to solve numerical problems involving integral equations and ordinary differential equations known as Sinc collocation methods. This method has been shown to be an efficient numerical tool for finding solution. The construction mixed strategies evolutionary game can be transformed to an integrodifferential problem. Properties of the sinc procedure are utilized to reduce the computation of this integrodifferential to some algebraic equations. The method is applied to a few test examples to illustrate the accuracy and implementation of the method.

\section{Introduction}

Evolutionary game dynamics is a fast developing field, with applications in biology, economics, sociology, politics, interpersonal relationships, and anthropology. Background material and countless references can be found in [1-8]. In the present paper we consider a continuous mixed strategies model for population dynamics based on an integrodifferential representation. Analogous models for population dynamics based on the replicator equation with continuous strategy space were investigated in [9-13]. For the moment based model has proved global existence of solutions and studied the asymptotic behavior and stability of solutions in the case of two strategies [14].

In the last three decades a variety of numerical methods based on the sinc approximation have been developed. Sinc methods were developed by Stenger [15] and Lund and Bowers [16] and it is widely used for solving a wide range of linear and nonlinear problems arising from scientific and engineering applications including oceanographic problems with boundary layers [17], two-point boundary value problems [18], astrophysics equations [19], Blasius equation [20], Volterras population model [21], Hallens integral equation [22], third-order boundary value problems [23], system of second-order boundary value problems [24], fourth-order boundary value problems [25], heat distribution [26], elastoplastic problem [27], inverse problem [28, 29], integrodifferential equation [30], optimal control [15], nonlinear boundary-value problems [31], and multipoint boundary value problems [32]. Very recently authors of [33] used the sinc procedure to solve linear and nonlinear Volterra integral and integrodifferential equations.

The content of this paper is arranged in seven sections. In Section 2, I discuss the modeling of the problem in an integrodifferential form. Section 3 , introduces some general concepts concerning the sinc approximation. Section 4 , contains some preliminaries in collocation method. In Section 5 , the method is applied for solving the problem. In Section 6, some numerical examples has been provided. Finally, Section 7 provides the conclusion of this work.

\section{Mathematical Model}

The model we consider here is an integrodifferential model for continuous mixed strategies. In game theory, a dominant strategy is the one that gives a player the most benefit no matter what the other players do. A player's strategy in a game is a complete plan of action for whatever situation might arise; this fully determines the player's behavior. A player's strategy 
set defines what strategies are available for them to play. A pure strategy provides a complete definition of how a player will play a game. In particular, it determines the move a player will make for any situation he or she could face. A player's strategy set is the set of pure strategies available to that player. A mixed strategy is an assignment of a probability to each pure strategy. This allows for a player to randomly select a pure strategy. Since probabilities are continuous, there are infinitely many mixed strategies available to a player, even if their strategy set is finite.

A payoff is a number, also called utility that reflects the desirability of an outcome to a player, for whatever reason. When the outcome is random, payoffs are usually weighted with their probabilities. The expected payoff incorporates the player's attitude towards risk.

Assume that we have a game where there are $N$ pure strategies $R_{1}$ to $R_{N}$ and that the players can use mixed strategies: this consists of playing the pure strategies $R_{1}$ to $R_{N}$ with some probabilities $q_{1}$ to $q_{N}$ with $q_{i} \geq 0$ and $\sum q_{i}=1$. A strategy corresponds to a point $\mathbf{q}$ in the simplex

$$
S_{N-1}=\left\{\mathbf{q}=\left(q_{1}, q_{2}, \ldots, q_{N}\right) \in R^{N}: q_{i} \geq 0, \sum_{i=1}^{N} q_{i}=1\right\} .
$$

The corners of the simplex are the standard unit vectors $\mathbf{e}_{i}$, where the $i$ th component is 1 and all others are 0 and correspond to the $N$ pure strategies $R_{i}, i=1, \ldots, N$.

Let us denote by $a_{i j}$ the payoff for a player using the pure strategy $R_{i}$ against a player using the pure strategy $R_{j}$. Here Matrix $A=\left(a_{i j}\right)$ is called payoff matrix. An $R_{i}$-strategist obtain the expected payoff $\left(A \mathbf{q}^{*}\right)_{i}=\sum_{j} a_{i j} q_{j}^{*}$ against a $\mathbf{q}^{*}$ strategist. The payoff for a $\mathbf{q}$-startegist against a $\mathbf{q}^{*}$ strategist is given by

$$
A\left(\mathbf{q}, \mathbf{q}^{*}\right):=\mathbf{q} \cdot A \mathbf{q}^{*}=\sum_{i, j=1}^{N} a_{i j} q_{i} q_{j}^{*}
$$

We consider a population of individuals as a player of the game and denote by $f(t, \mathbf{q})$ the density of population adopting the $\mathbf{q}$ strategy at time $t$; the evolution in time of $f$, due to dynamics of the game, is driven by

$$
\partial_{t} f(t, \mathbf{q})=f(t, \mathbf{q})\left(\int_{S_{N}} A\left(\mathbf{q}, \mathbf{q}^{*}\right) f\left(t, \mathbf{q}^{*}\right) d \mathbf{q}^{*}-\phi(f)\right),
$$

where the term

$$
\int_{S_{N}} A\left(\mathbf{q}, \mathbf{q}^{*}\right) f\left(t, \mathbf{q}^{*}\right) d \mathbf{q}^{*},
$$

represents the payoff of the strategy $\mathbf{q}$ against all the others strategies, $A\left(\mathbf{q}, \mathbf{q}^{*}\right)$ being the interacting kernel between the q-strategist and the $\mathbf{q}^{*}$-strategist. The last term of (3) is defined by

$$
\phi(f):=\iint_{S_{N}} f(t, \mathbf{q}) A\left(\mathbf{q}, \mathbf{q}^{*}\right) f\left(t, \mathbf{q}^{*}\right) d \mathbf{q}^{*} d \mathbf{q}
$$

and represents the average payoff of the population.
Since $\sum_{i=1}^{N} q_{i}=1$, we can reduce the number of variables, considering

$$
q_{N}=1-\sum_{i=1}^{N-1} q_{i}
$$

and obtaining the $(N-1)$-dimensional model (3) on the simplex

$$
\tau_{N-1}:=\left\{\mathbf{p}=\left(p_{1}, p_{2}, \ldots, p_{N-1}\right) \in R^{N-1}: p_{i} \geq 0, \sum_{i=1}^{N-1} p_{i} \leq 1\right\},
$$

namely,

$$
\partial_{t} f(t, \mathbf{p})=f(t, \mathbf{p})\left(\int_{\tau_{N-1}} A\left(\mathbf{p}, \mathbf{p}^{*}\right) f\left(t, \mathbf{p}^{*}\right) d p^{*}-\phi(f)\right),
$$

with $A\left(\mathbf{p}, \mathbf{p}^{*}\right)$ defined by

$$
A\left(\mathbf{p}, \mathbf{p}^{*}\right):=\mathbf{p} \cdot A p^{*}=\sum_{i, j=1}^{N-1} a_{i j} p_{i} p_{j}^{*}
$$

and $\phi$ defined by

$$
\phi(f):=\iint_{\tau_{N-1}} f(t, \mathbf{p}) A\left(\mathbf{p}, \mathbf{p}^{*}\right) f\left(t, \mathbf{p}^{*}\right) d \mathbf{p}^{*} d \mathbf{p} .
$$

Remark 1 (see [14]). If we take an initial condition

$$
f(0, \mathbf{p})=f_{0}(\mathbf{p}) \geq 0,
$$

with $\int_{N-1} f_{0}(\mathbf{p}) d \mathbf{p}=1$, then it is easy to see that $f \geq 0$ for all $t>0$ and if $f_{0}(\overline{\mathbf{p}})=0$ for some $\overline{\mathbf{p}}$, then $f(t, \overline{\mathbf{p}})=0$ for all $t>0$. We also know that

$$
\int_{\tau_{N-1}} f(t, \mathbf{p}) d \mathbf{p}=1, \quad \forall t>0 .
$$

This follows from the mass conservation; by integrating (8) with respect to $\mathbf{p}$ and using (10) and (12) we have

$$
\partial_{t} \int_{\tau_{N-1}} f(t, \mathbf{p}) d \mathbf{p}=0
$$

Let us introduce the moments for $f$ :

$$
M_{k}(f):=\int_{\tau_{N-1}} \mathbf{p}^{k} f(\mathbf{p}) d \mathbf{p}=\int_{\tau_{N-1}} p_{1}^{k_{1}} p_{2}^{k_{2}} \ldots p_{N-1}^{k_{N-1}} f(\mathbf{p}) d \mathbf{p}
$$

with $\mathbf{k}:=\left(k_{1}, k_{2}, \ldots, k_{N-1}\right)$. Using $M_{k}(f)$, the payoff and the average payoff (10)

$$
\begin{aligned}
\int_{\tau_{N-1}} A\left(\mathbf{p}, \mathbf{p}^{*}\right) f\left(t, \mathbf{p}^{*}\right) d \mathbf{p}^{*} \\
=\sum_{j=1}^{N-1} M_{e_{j}}(f)\left(\sum_{i=1}^{N-1} \vartheta_{i, j} p_{i}+\varsigma_{j}\right)+a_{N, N}+\sum_{i=1}^{N-1} v_{i} p_{i},
\end{aligned}
$$




$$
\begin{aligned}
\phi(f)= & \sum_{j=1}^{N-1} M_{e_{j}}(f)\left(\sum_{i=1}^{N-1} \vartheta_{i, j} M_{e_{j}}(f)+\varsigma_{j}\right) \\
& +a_{N, N}+\sum_{i=1}^{N-1} v_{i} M_{e_{i}}(f),
\end{aligned}
$$

where $e_{i} \in R^{N-1}$ is the standard unit vector with the $i$ th component equal to 1 and all others equal to 0 . Moreover, $\vartheta_{i, j}:=a_{i, j}-a_{i, N}-a_{N, j}+a_{N, N}, \varsigma_{j}:=a_{N, j}-a_{N, N}, v_{i}:=a_{i, N}-a_{N, N}$.

In the final form of (8), that will be used later in this paper, the only integral terms are the first moments $M_{e_{i}}$ :

$$
\begin{aligned}
\partial_{t} f & (t, \mathbf{p}) \\
& =f(t, \mathbf{p})\left(\sum_{i=1}^{N-1}\left(p_{i}-M_{e_{i}}(f)\right)\left(v_{i}+\sum_{i=1}^{N-1} \vartheta_{i, j} M_{e_{j}}(f)\right)\right) .
\end{aligned}
$$

Global Existence of the Solutions. We consider the Cauchy problem (11)-(16) for $t \geq 0$ and $p \in \tau_{N-1}$; that is,

$$
\begin{aligned}
\partial_{t} f(t, \mathbf{p})= & f(t, \mathbf{p})\left(\sum_{i=1}^{N-1}\left(p_{i}-M_{e_{i}}(f)\right)\right. \\
& \left.\times\left(v_{i}+\sum_{i=1}^{N-1} \vartheta_{i, j} M_{e_{j}}(f)\right)\right), \\
& f(0, \mathbf{p})=f_{0}(\mathbf{p})
\end{aligned}
$$

with $f_{0}(p) \geq 0$ and $\int_{\tau_{N-1}} f(t, \mathbf{p}) d \mathbf{p}=1$.

Proposition 2 (local existence see [14]). For all $M>0$ there exists $T(M)>0$ such that if $\left\|f_{0}(\mathbf{p})\right\| \leq M$; then there exists a unique solution $f \in C\left([0, \widetilde{T}] \times \tau_{N-1}\right)$ for the problem (17), for all $\widetilde{T} \leq T(M)$.

2.1. Two Strategies Games. Assume there are two different strategies, whose interplay is ruled by the payoff matrix:

$$
A=\left(\begin{array}{ll}
a & b \\
c & d
\end{array}\right)
$$

In this case the simplex $\tau_{1}$ is just the interval $[0,1]$ and so we have a population where individuals are going to play the first strategy with probability $p \in[0,1]$ and the second strategy with probability $1-p$. The payoff (2) is given by

$$
\begin{aligned}
A\left(\mathbf{p}, \mathbf{p}^{*}\right) & :=\left(\begin{array}{c}
p \\
1-p
\end{array}\right)\left(\begin{array}{ll}
a & b \\
c & d
\end{array}\right)\left(\begin{array}{c}
p^{*} \\
1-p^{*}
\end{array}\right) \\
& =(a+d-b-c) p p^{*}+(b-d) p+(c-d) p^{*}+d \\
& =\alpha p p^{*}+\beta p+\gamma p^{*}+\delta
\end{aligned}
$$

with

$$
\begin{aligned}
& \alpha:=(a+d-b-c), \\
& \beta:=b-d, \\
& \gamma:=c-d, \\
& \delta:=d .
\end{aligned}
$$

The one dimensional Cauchy problem (17) reads

$$
\begin{gathered}
\partial_{t} f(p)=f(p)\left(\alpha M_{1}(f)+\beta\right)\left(p-M_{1}(f)\right) \\
t \geq 0, \quad p \in[0,1] \\
f(0, p)=f_{0}(p) \quad p \in[0,1]
\end{gathered}
$$

with $f_{0}(p) \geq 0$ and $\int_{0}^{1} f_{0}(p) d p=1$.

For more detail see [14].

\section{Sinc Interpolation}

The goal of this section is to recall notations and definition of the sinc function that are used. The sinc approximation for a function $f(x)$ defined on the real line $R$ is given by

$$
f(x) \approx \sum_{j=-N}^{N} f(j h) S(j, h)(x)
$$

where $S(j, h)$ is sinc function defined by

$$
S(j, h)(x):=\frac{\sin [(\pi / h)(x-j h)]}{[(\pi / h)(x-j h)]} .
$$

And the step size $h$ is suitably chosen for a given positive integer $n=2 N+1$. Sinc for interpolation points $x_{k}=k h$ is given by

$$
S(j, h)(k h)=\delta_{j k}^{(0)}= \begin{cases}1 & k=j, \\ 0 & k \neq j .\end{cases}
$$

Assuming that $f(t)$ is analytic on the real line and decays exponentially on the real line, it has been shown that the error of the approximation decays exponentially with increasing $N$. The approximation may be extended to approximate $f(t)$ on the interval $[0,1]$ by selection of an appropriate transfer function to transform the interval onto the real line and impose the exponential decay. We denote such variable transformation $\tau=\phi(t)$ and inverse transformation $t=$ $\psi(\tau)$ such that $\phi(0)=-\infty$ and $\phi(1)=\infty$. We may write the sinc approximation employing the transformation for the function $f(t)$ to be

$$
f(x) \approx \sum_{j=-N}^{N} f(\psi(j h)) S(j, h)(x)(\phi(t)),
$$

where the mesh size $h$ represents the separation between sinc points on the $\tau \in(-\infty, \infty)$ domain. In order to have the 
sinc approximation on a finite interval $(0,1)$ conformal map is employed as follows:

$$
\phi(z)=\ln \left(\frac{z}{1-z}\right) .
$$

This map carries the eye-shaped complex domain

$$
D_{E}=\left\{z=x+i y:\left|\arg \left(\frac{z}{1-z}\right)\right|<d \leq \frac{\pi}{2}\right\},
$$

onto the infinite strip

$$
D_{s}=\left\{w=u+i v:|v|<d \leq \frac{\pi}{2}\right\} .
$$

For the sinc method, the basic function on the interval $(0,1)$ for $z \in D_{E}$ is derived from the composite translated sinc functions:

$$
S_{j}(z)=s(j, h) o \phi(z)=\operatorname{Sinc}\left(\frac{\phi(z)-j h}{h}\right) .
$$

Exhibiting kroneckor delta behavior on the grid points

$$
x_{k}=\phi^{-1}(k h)=\frac{e^{k h}}{1+e^{k h}}, \quad k= \pm 1, \pm 2, \ldots
$$

Thus we may define the inverse images of the real line and of the evenly space nodes $\{k h\}_{k=-\infty}^{\infty}$ as

$$
\Gamma=\left\{\phi^{-1}(x) \in D_{E}:-\infty<x<\infty\right\}=(0,1) .
$$

And quadrature formulas for $f(t)$ over $[0,1]$ are

$$
\begin{gathered}
f(x) \approx \sum_{k=-N}^{N} f\left(x_{k}\right) S(k, h) o \phi(x), \\
\int_{0}^{1} f(x) d x \approx h \sum_{k=-N}^{N} \frac{f\left(x_{k}\right)}{\phi^{\prime}\left(x_{k}\right)} .
\end{gathered}
$$

Definition 3. Let $B\left(D_{E}\right)$ be the class of functions $F$ which are analytic in $D_{E}$ satisfy

$$
\int_{\psi(t+L)}|F(z) d z| \longrightarrow 0, \quad t \longrightarrow \pm \infty
$$

where, $L=\{i v:|v|<d \leq \pi / 2\}$ and on the boundary of $D_{E}$ (denoted by $\partial D_{E}$ ) satisfying

$$
N(F)=\int_{\partial D_{E}}|F(z) d z|<\infty .
$$

Interpolation for function in $B\left(D_{E}\right)$ is defined in the following theorem whose proof can be found in [15].

Theorem 4. If $\phi^{\prime} F \in B\left(D_{E}\right)$ then for all $x \in \Gamma$

$$
\begin{aligned}
\left|F(x)-\sum_{k=-\infty}^{\infty} F\left(x_{k}\right) S(k, h) o \phi(x)\right| & \leq \frac{N\left(F \phi^{\prime}\right)}{2 \pi d \sinh (\pi d / h)} \\
& \leq \frac{2 N\left(F \phi^{\prime}\right)}{\pi d} e^{-\pi d / h} .
\end{aligned}
$$

Moreover, if $|F(x)| \leq C e^{-\alpha|\phi(x)|}, x \in \Gamma$ for some positive constants $C$ and $\alpha$, and if the selection $h=\sqrt{\pi d / \alpha N} \leq$ $2 \pi d / \ln 2$ then

$$
\begin{aligned}
& \left|F(x)-\sum_{k=-N}^{N} F\left(x_{k}\right) S(k, h) o \phi(x)\right| \\
& \leq C_{2} \sqrt{N} \exp (-\sqrt{\pi d \alpha N}), \quad x \in \Gamma,
\end{aligned}
$$

where, $C_{2}$ depends only on $F, d$, and $\alpha$. The above expressions show sinc interpolation on $B\left(D_{E}\right)$ converge exponentially [17]. We also require derivatives of composite sinc functions evaluated at the nodes. The expressions required for the present discussion are [25]

$$
\begin{gathered}
\delta_{k, j}^{(0)}=[S(k, h) o \phi(x)]_{x=x_{j}}= \begin{cases}1 & k=j, \\
0 & k \neq j,\end{cases} \\
\delta_{k, j}^{(1)}=\frac{d}{d \phi}[S(k, h) o \phi(x)]_{x=x_{j}}=\frac{1}{h} \begin{cases}0 & k=j, \\
\frac{(-1)^{j-k}}{j-k} & k \neq j,\end{cases} \\
\delta_{k, j}^{(2)}=\frac{d^{2}}{d \phi^{2}}[S(k, h) o \phi(x)]_{x=x_{j}}=\frac{1}{h^{2}} \begin{cases}\frac{-\pi^{2}}{3} & k=j, \\
\frac{-2(-1)^{j-k}}{(j-k)^{2}} & k \neq j .\end{cases}
\end{gathered}
$$

\section{Collocation Method}

Let $I_{h}=\left\{t_{n}: 0=t_{0}<t_{1}<\cdots<t_{N}=T\right\}$ be a given mesh (not necessarily uniform) on $I$ and set $\sigma_{n}:=\left(t_{n}, t_{n+1}\right]$, $\bar{\sigma}_{n}:=\left[t_{n}, \mathrm{t}_{n+1}\right]$, with $h_{n}:=t_{n+1}-t_{n}(n=0,1, \ldots, N-1)$. The quantity $h:=\max \left\{h_{n}: 0 \leq n \leq N-1\right\}$ will be called the diameter of the mesh $I_{h}$.

Definition 5. Suppose that $I_{h}$ is a given partition on $I$. The piecewise polynomials space $S_{\mu}^{(d)}\left(I_{h}\right)$ with $\mu \geq 0,-1 \leq d \leq \mu$ is defined by

$$
S_{\mu}^{(d)}\left(I_{h}\right)=\left\{q(t) \in C^{d}[I, R]:\left.q\right|_{\sigma_{n}} \in \pi_{\mu} ; 0 \leq n \leq N-1\right\} .
$$

Here $\sigma_{n}=\left(t_{n}, t_{n+1}\right]$ and $\pi_{\mu}$ denote the space of polynomials of degree not exceeding $\mu$, and it is easy to see that $S_{\mu}^{(d)}\left(I_{h}\right)$

$$
S_{\mu}^{(d)}\left(I_{h}\right)=N(\mu-d)+d+1 .
$$

The collocation solution is determined by $u_{h}$ that satisfies the given equation on a given suitable finite subset $X_{h}$ of $I$, where $X_{h}$ contains the collocation points:

$$
X_{h}=\left\{t_{n}+c_{i} h_{n}: 0 \leq c_{1} \leq \cdots \leq c_{m} \leq 1 ; 0 \leq n \leq N-1\right\}
$$

is determined by the points of the partition $I_{h}$ and the given collocation parameters $\left\{c_{i}\right\} \in[0,1]$. The collocation solution $u_{h} \in S_{m}^{(0)}\left(I_{h}\right)$ for

$$
\dot{y}(t)=f(t, y(t)) \quad y(0)=y_{0}
$$


is defined by the collocation equation

$$
\dot{u}_{h}(t)=f\left(t, u_{h}(t)\right), \quad t \in X_{h}, u_{h}(0)=y_{0} .
$$

It will be convenient (and natural) to work with the local Lagrange basis representations of $u_{h}$. These polynomials in $\sigma_{n}$ can be written as

$$
L_{j}(z)=\Pi_{k \neq j}^{m} \frac{\left(z-c_{k}\right)}{\left(c_{j}-c_{k}\right)}, \quad z \in[0,1], j=1, \ldots, m,
$$

where $L_{j}(z)$ belong to $\pi_{m-1}$. Also we have

$$
\begin{array}{r}
u_{h}\left(t_{n}+v h_{n}\right)=\sum_{j=1}^{m} L_{j}(v) Y_{n, j}, \quad v \in(0,1], \\
Y_{n, j}:=u_{h}\left(t_{n}+c_{j} h_{n}\right) .
\end{array}
$$

From (44) we can obtain the local representation of $u_{h} \epsilon$ $S_{m}^{(0)}\left(I_{h}\right)$ on $\bar{\sigma}_{n}$, hence we can achieve that

$$
u_{h}^{\prime}\left(t_{n}+v h_{n}\right)=\frac{1}{h_{n}} \sum_{j=1}^{m} L_{j}^{\prime}(v) Y_{n, j}
$$

The unknown approximations $Y_{n, i}(i=1, \ldots, m)$ in $(44)$ are defined by the solution of a system of (generally nonlinear) algebraic equations obtained by setting $t=t_{n, i}:=t_{n}+c_{i} h_{n}$ in the collocation equation (42) and employing the local representation (44). This system is

$$
\frac{1}{h_{n}} \sum_{j=1}^{m} L_{j}^{\prime}\left(c_{i}\right) Y_{n, j}=f\left(t_{n, i}, Y_{n, i}\right), \quad i=1, \ldots, m
$$

It corresponds to (44) with $v=1$,

$$
y_{n+1}:=u_{h}\left(t_{n}+h_{n}\right)=\sum_{j=1}^{m} L_{j}(1) Y_{n, j} \quad(n=0,1, \ldots, N-1) .
$$

I present the result on global convergence for the linear initial-value problem

$$
\dot{y}(t)=a(t) y(t)+g(t), \quad t \in I, \quad y(0)=y_{0} .
$$

Theorem 6. Assume that

(a) the given functions in (48) satisfy a, $g \in C^{m}(I)$;

(b) $\bar{h}>0$ is such that, for any $h \in(0, \bar{h})$, each of the linear systems of method has a unique solution.

Then the estimates

$$
\begin{aligned}
& \left\|y-u_{h}\right\|_{\infty}:=\max _{t \in I}\left|y(t)-u_{h}(t)\right| \leq C_{0}\left\|y^{(m+1)}\right\|_{\infty} h^{m}, \\
& \left\|\dot{y}-\dot{u}_{h}\right\|_{\infty}:=\max _{t \in I}\left|y(t)-\dot{u}_{h}(t)\right| \leq C_{1}\left\|y^{(m+1)}\right\|_{\infty} h^{m} .
\end{aligned}
$$

See [34].

\section{Construction of the Method}

Let $\left\{0=t_{0}<t_{1}<\cdots<t_{N}=T\right\}$ is a partition of $[0, T]$. In every interval $\sigma_{n}=\left(t_{n}, t_{n+1}\right]$ we assume that $f(t, p), p \in$ $[0,1]$, solution of one dimensional mixed strategy model is approximated by the finite expansion of sinc basis function and Lagrange polynomials:

$$
f(t, p) \simeq \sum_{j=0}^{m} \sum_{k=-N}^{N} c_{j, k} S(k, h) o \phi(p) \Psi_{j}(t),
$$

where, $\Psi_{j}(t)$ is a polynomial of degree $m$. Also, initial value is according to

$$
f\left(t_{n}, p\right)=f_{t_{n}}(p)
$$

If we replace approximation (51) in (21) we have

$$
\begin{aligned}
& \sum_{j=0}^{m} \sum_{k=-N}^{N} c_{j, k} S(k, h) o \phi(p) \Psi_{j}^{\prime}(t) \\
& =\sum_{j=0}^{m} \sum_{k=-N}^{N} c_{j, k} S(k, h) o \phi(p) \Psi_{j}(t) \\
& \quad \times\left(\alpha M_{1}(f(t, \mathrm{p}))+\beta\right)\left(p-M_{1}(f(t, p))\right),
\end{aligned}
$$

where $M_{1}(f)$ is taken by

$$
M_{1}(f(t, p))=\sum_{j=0}^{m} \sum_{k=-N}^{N} c_{j, k} \int_{0}^{1} p \cdot S(k, h) o \phi(p) \Psi_{j}(t) d p .
$$

By substituting collocation points for $t$ and $p$ and using quadrature rule (32), a nonlinear system is given.

After solving this system we calculate $c_{j, k}$ and finally $f(t, p)$ in $\sigma_{n}$, and also, $f(t, p)$ at $t_{n+1}$ :

$$
f\left(t_{n+1}, p\right)=\sum_{j=0}^{m} \sum_{k=-N}^{N} c_{j, k} S(k, h) o \phi(p) \Psi_{j}\left(t_{n+1}\right),
$$

where $f\left(t_{n+1}, p\right)$ is used as an initial value for next interval $\sigma_{n+1}$. After $N$ times, solution is achieved.

\section{Numerical Examples}

Prisoner's Dilemma Game. One interesting example of a game is given by the so-called Prisoner's Dilemma game in which there are two players and two possible strategies. The players have two options, cooperate or defect. The payoff matrix is the following:

$$
A=\left(\begin{array}{ll}
R & S \\
T & P
\end{array}\right)
$$

If both players cooperate both obtain $R$ fitness units (reward payoff); if both defect, each receives $P$ (punishment payoff); if one player cooperates and the other defects, the cooperator 


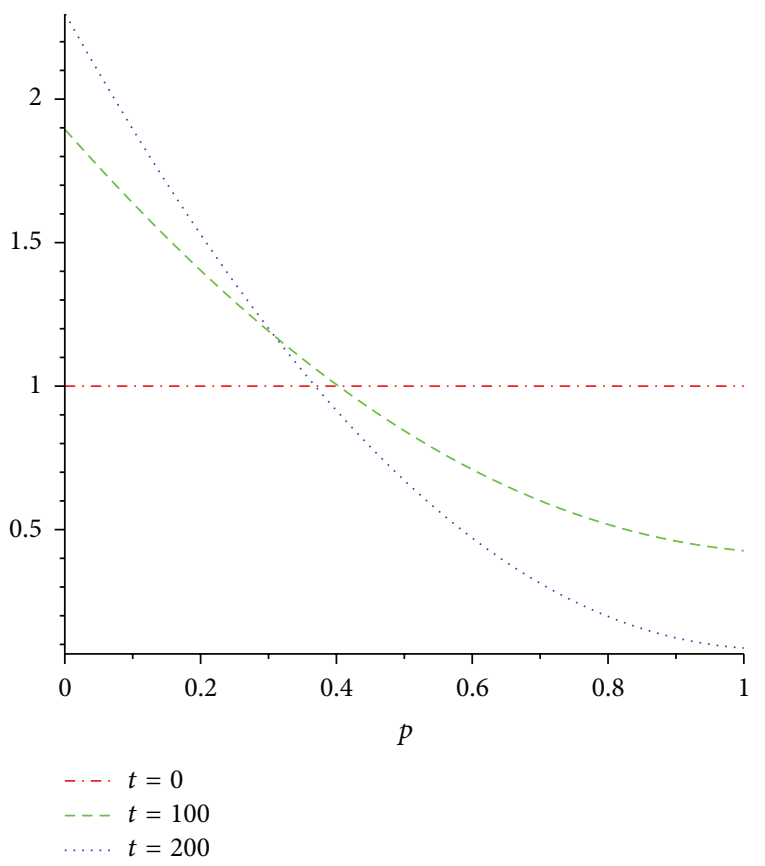

Figure 1: Plot of the evolution over time of $f(t, p)$ for the Prisoner's Dilemma game with $f_{0}(p)=1$.

gets $S$ (suckers payoff) while the defector gets $T$ (temptation payoff). The payoff values are ranked $T>R>P>S$ and $2 R>T+S$. We know that cooperators are always dominated by defectors.

For the numerical tests we fix the following normalized payoff matrix:

$$
A=\left(\begin{array}{ll}
1 & 0 \\
b & \epsilon
\end{array}\right)
$$

with $b=1.1$ and $\epsilon=0.001$. In this case we have $\alpha=1-$ $b+\epsilon<0$ and $\beta=-\epsilon<0$ and so $\beta / \alpha>0$. This means that stationary solutions are expected to be given by concentrated Dirac masses. For general perturbation we have that $\bar{p}=0$ is linearly stable.

We In order to conform the results above, initial condition is considered as below:

(1)

$$
f_{0}(p)=1, \quad \forall p \in[0,1]
$$

(2)

$$
f_{0}(p)=-p^{2}+\frac{2}{3} p+1, \quad \forall p \in[0,1]
$$

(3)

$$
f_{0}(p)= \begin{cases}2 & p \in\left[\frac{1}{4}, \frac{1}{2}\right] \cup\left[\frac{3}{4}, 1\right], \\ 0 & \text { elsewhere }\end{cases}
$$

For implementation of proposed method, I used Maple15 and plotted the numerical results in Figures 1, 2, and 3. Figure 1 shows that the density $f$ tends to concentrate at the point $p=$ 0 , to what we expected.

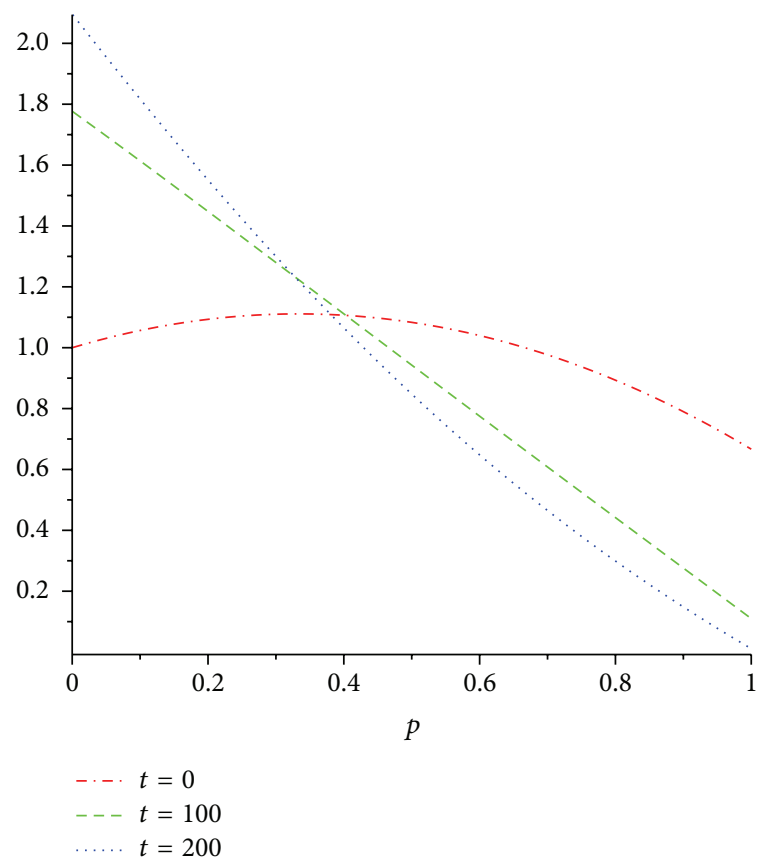

Figure 2: Plot of the evolution over time of $f(t, p)$ for the Prisoner's Dilemma game with $f_{0}(p)=-p^{2}+(2 / 3) p+1$.

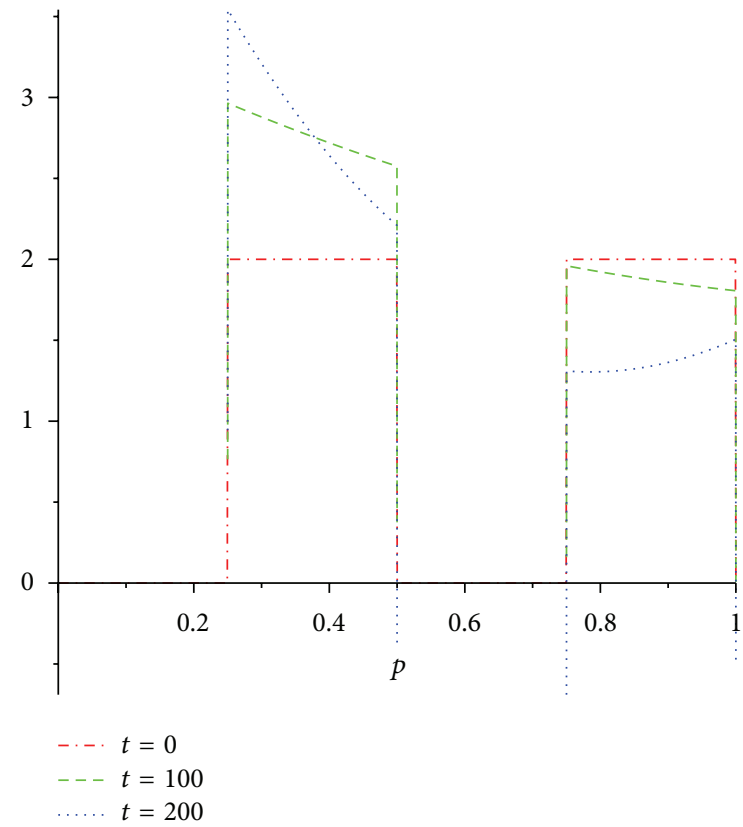

Figure 3: Plot of the evolution over time of $f(t, p)$ for the Prisoner's Dilemma game with $f_{0}(p)=\left\{\begin{array}{cc}2 & p \in[1 / 4,1 / 2] \cup[3 / 4,1], \\ 0 & \text { elsewhere. }\end{array}\right.$.

\section{Conclusion}

In this paper, the collocation method with sinc and Lagrange polynomials are employed to construct an approximation to the solution of continuous mixed strategy. It is found that the results of the present works agree well with trapezoidal rule. Properties of the sinc procedure are utilized to reduce 
the computation of this integrodifferential to some nonlinear equations. There are several advantages over classical methods to using approximations based on sinc numerical methods. First, unlike most numerical techniques, it is now well-established that they are characterized by exponentially decaying errors. Secondly, approximation by sinc functions handles singularities in the problem. Thirdly, due to their rapid convergence, sinc numerical methods do not suffer from the common instability problems associated with other numerical methods. Also, in this case the advantages of collocation method are used. The method is applied to test examples to illustrate the accuracy and implementation of the method.

\section{Conflict of Interests}

The author declares that there is no conflict of interests regarding the publication of this paper.

\section{References}

[1] J. W. Weibull, Evolutionary Game Theory, The MIT Press, 1995.

[2] D. Fudenberg and D. Levine, The Theory of Learning in Games, MIT Press, 1998.

[3] L. Samuelson, Evolutionary Games and Equilibrium Selection, MIT Press, 1998.

[4] J. Hofbauer and K. Sigmund, Evolutionary Games and Population Dynamics, Cambridge University Press, Cambridge, UK, 1998.

[5] H. Gintis, Game Theory Evolving, Princeton University Press, 2000.

[6] R. Cressman, Evolutionary Dynamics and Extensive Form Games, MIT Press, 2003.

[7] Th. Vincent and J. Brown, Evolutionary Game Theory, Natural Selection and Darwinian Dynamics, Cambridge University Press, Cambridge, UK, 2005.

[8] J. Hofbauer and K. Sigmund, "Evolutionary game dynamics," Bulletin of the American Mathematical Society, vol. 40, no. 4, pp. 479-519, 2003.

[9] I. M. Bomze, "Dynamical aspects of evolutionary stability," Monatshefte für Mathematik, vol. 110, no. 3-4, pp. 189-206, 1990.

[10] R. Cressman, "Stability of the replicator equation with continuous strategy space," Mathematical Social Sciences, vol. 50, no. 2 , pp. 127-147, 2005.

[11] J. Hofbauer, J. Oechssler, and F. Riedel, "Brown-von NeumannNASh dynamics: the continuous strategy case," Games and Economic Behavior, vol. 65, no. 2, pp. 406-429, 2009.

[12] T. W. L. Norman, "Dynamically stable sets in infinite strategy spaces," Games and Economic Behavior, vol. 62, no. 2, pp. 610627, 2008.

[13] J. Oechssler and F. Riedel, "Evolutionary dynamics on infinite strategy spaces," Economic Theory, vol. 17, no. 1, pp. 141-162, 2001.

[14] A. Boccabella, R. Natalini, and L. Pareschi, "On a continuous mixed strategies model for evolutionary game theory," Kinetic and Related Models, vol. 4, no. 1, pp. 187-213, 2011.

[15] F. Stenger, Numerical Methods Based on Sinc and Analytic Functions, Springer, New York, NY, USA, 1993.

[16] J. Lund and K. L. Bowers, Sinc Methods for Quadrature and Differential Equations, SIAM, Philadelphia, Pennsylvania, USA, 1992.
[17] D. F. Winter, K. L. Bowers, and J. Lund, "Wind-driven currents in a sea with a variable eddy viscosity calculated via a SincGalerkin technique," International Journal for Numerical Methods in Fluids, vol. 33, no. 7, pp. 1041-1073, 2000.

[18] B. Bialecki, "Sinc-collocation methods for two-point boundary value problems," IMA Journal of Numerical Analysis, vol. 11, no. 3, pp. 357-375, 1991.

[19] K. Parand and A. Pirkhedri, "Sinc-Collocation method for solving astrophysics equations," New Astronomy, vol. 15, no. 6, pp. 533-537, 2010.

[20] K. Parand, M. Dehghan, and A. Pirkhedri, "Sinc-collocation method for solving the Blasius equation," Physics Letters A, vol. 373, no. 44, pp. 4060-4065, 2009.

[21] K. Parand, Z. Delafkar, N. Pakniat, A. Pirkhedri, and M. K. Haji, "Collocation method using sinc and rational Legendre functions for solving Volterra's population model," Communications in Nonlinear Science and Numerical Simulation, vol. 16, no. 4, pp. 1811-1819, 2011.

[22] A. Saadatmandi, M. Razzaghi, and M. Dehghan, "Sinc-collocation methods for the solution of Hallen's integral equation," Journal of Electromagnetic Waves and Applications, vol. 19, no. 2, pp. 245-256, 2005.

[23] A. Saadatmandi and M. Razzaghi, "The numerical solution of third-order boundary value problems using sinc-collocation method," Communications in Numerical Methods in Engineering, vol. 23, no. 7, pp. 681-689, 2007.

[24] M. Dehghan and A. Saadatmandi, "The numerical solution of a nonlinear system of second-order boundary value problems using the sinc-collocation method," Mathematical and Computer Modelling, vol. 46, no. 11-12, pp. 1434-1441, 2007.

[25] M. El-Gamel, S. H. Behiry, and H. Hashish, "Numerical method for the solution of special nonlinear fourth-order boundary value problems," Applied Mathematics and Computation, vol. 145, no. 2-3, pp. 717-734, 2003.

[26] P. N. Dinh Alain, P. H. Quan, and D. D. Trong, "Sinc approximation of the heat distribution on the boundary of a twodimensional finite slab," Nonlinear Analysis: Real World Applications, vol. 9, no. 3, pp. 1103-1111, 2008.

[27] K. Abdella, X. Yu, and I. Kucuk, "Application of the Sinc method to a dynamic elasto-plastic problem," Journal of Computational and Applied Mathematics, vol. 223, no. 2, pp. 626-645, 2009.

[28] J. Lund and C. R. Vogel, "A fully-Galerkin method for the numerical solution of an inverse problem in a parabolic partial differential equation," Inverse Problems, vol. 6, no. 2, pp. 205-217, 1990.

[29] A. Shidfar, R. Zolfaghari, and J. Damirchi, "Application of sinccollocation method for solving an inverse problem," Journal of Computational and Applied Mathematics, vol. 233, no. 2, pp. 545-554, 2009.

[30] J. Rashidinia and M. Zarebnia, "The numerical solution of integro-differential equation by means of the sinc method," Applied Mathematics and Computation, vol. 188, no. 2, pp. 11241130, 2007.

[31] M. El-Gamel and A. I. Zayed, "Sinc-Galerkin method for solving nonlinear boundary-value problems," Computers \& Mathematics with Applications, vol. 48, no. 9, pp. 1285-1298, 2004.

[32] A. Saadatmandi and M. Dehghan, "The use of sinc-collocation method for solving multi-point boundary value problems," Communications in Nonlinear Science and Numerical Simulation, vol. 17, no. 2, pp. 593-601, 2012. 
[33] A. Mohsen and M. El-Gamel, "On the numerical solution of linear and nonlinear Volterra integral and integro-differential equations," Applied Mathematics and Computation, vol. 217, no. 7, pp. 3330-3337, 2010.

[34] H. Brunner, Collocation Methods for Volterra Integral and Related Functional Differential Equations, Cambridge University Press, 2004. 

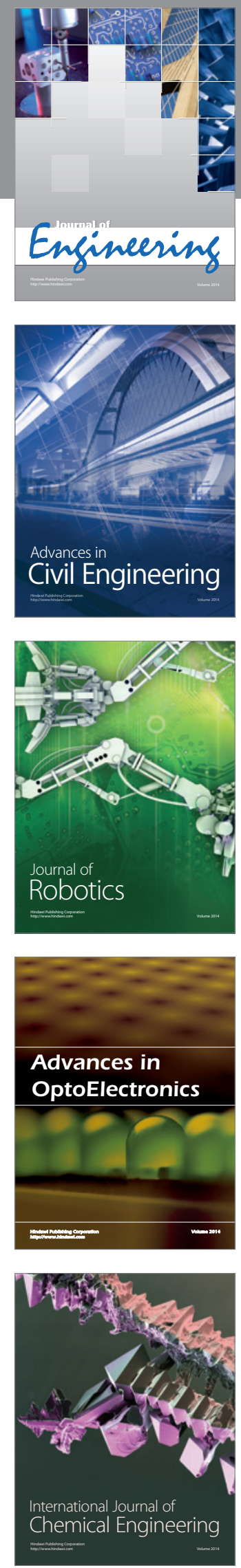

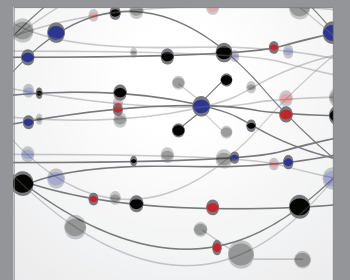

The Scientific World Journal
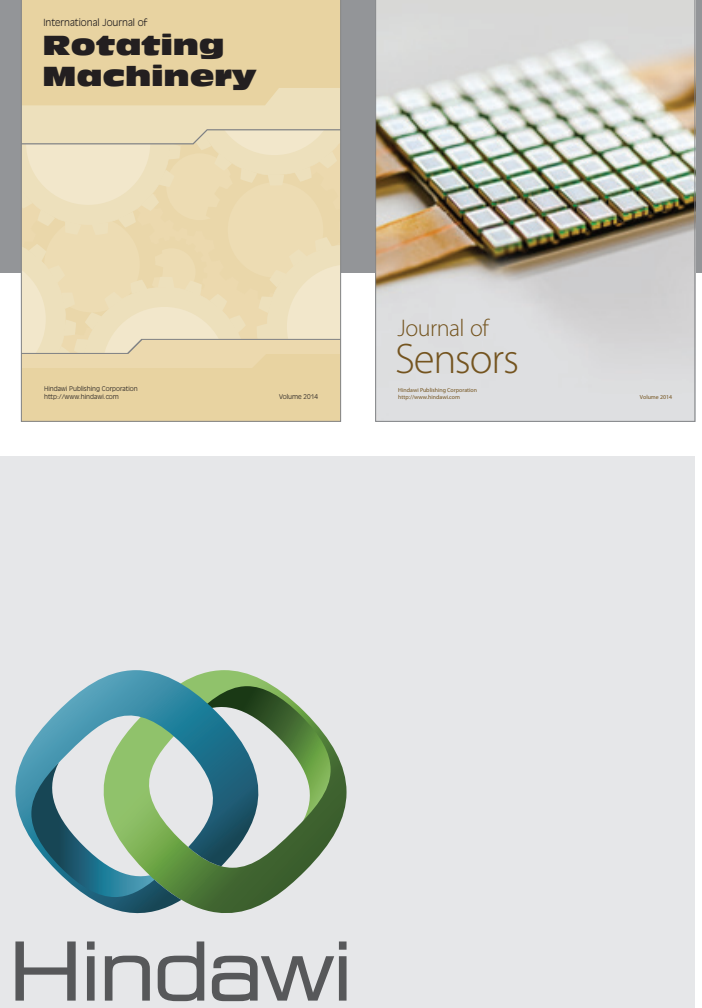

Submit your manuscripts at http://www.hindawi.com
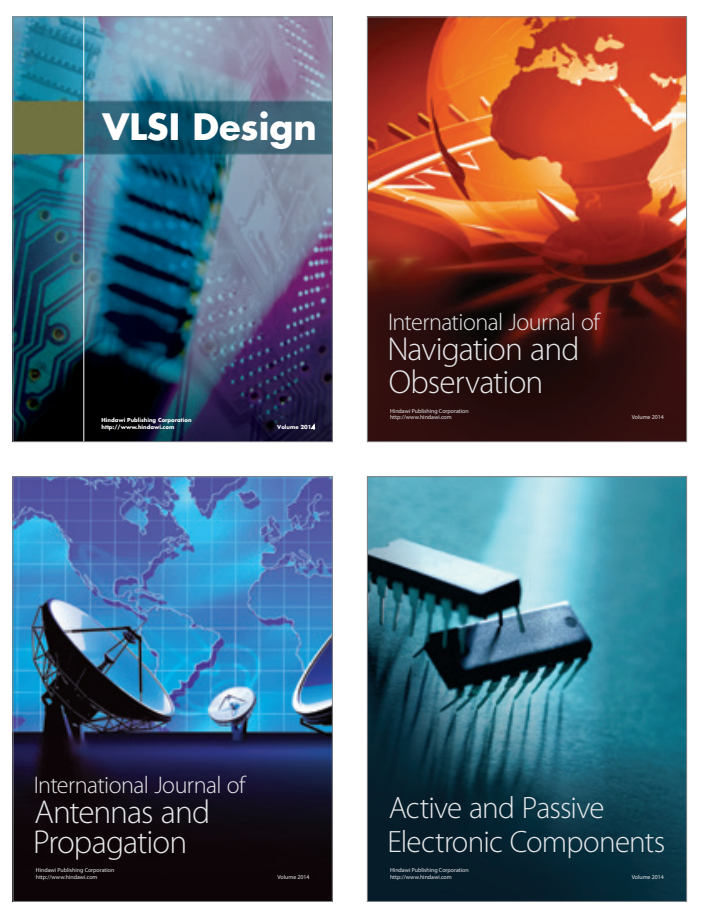
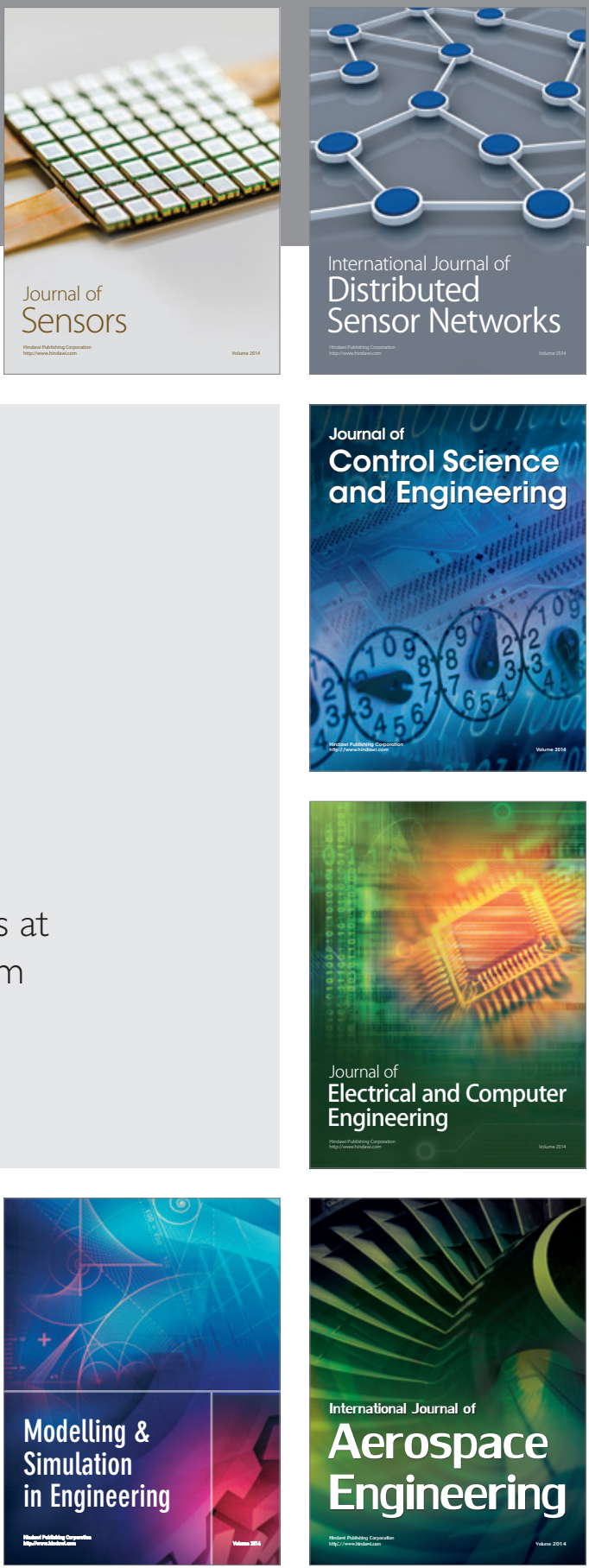

Journal of

Control Science

and Engineering
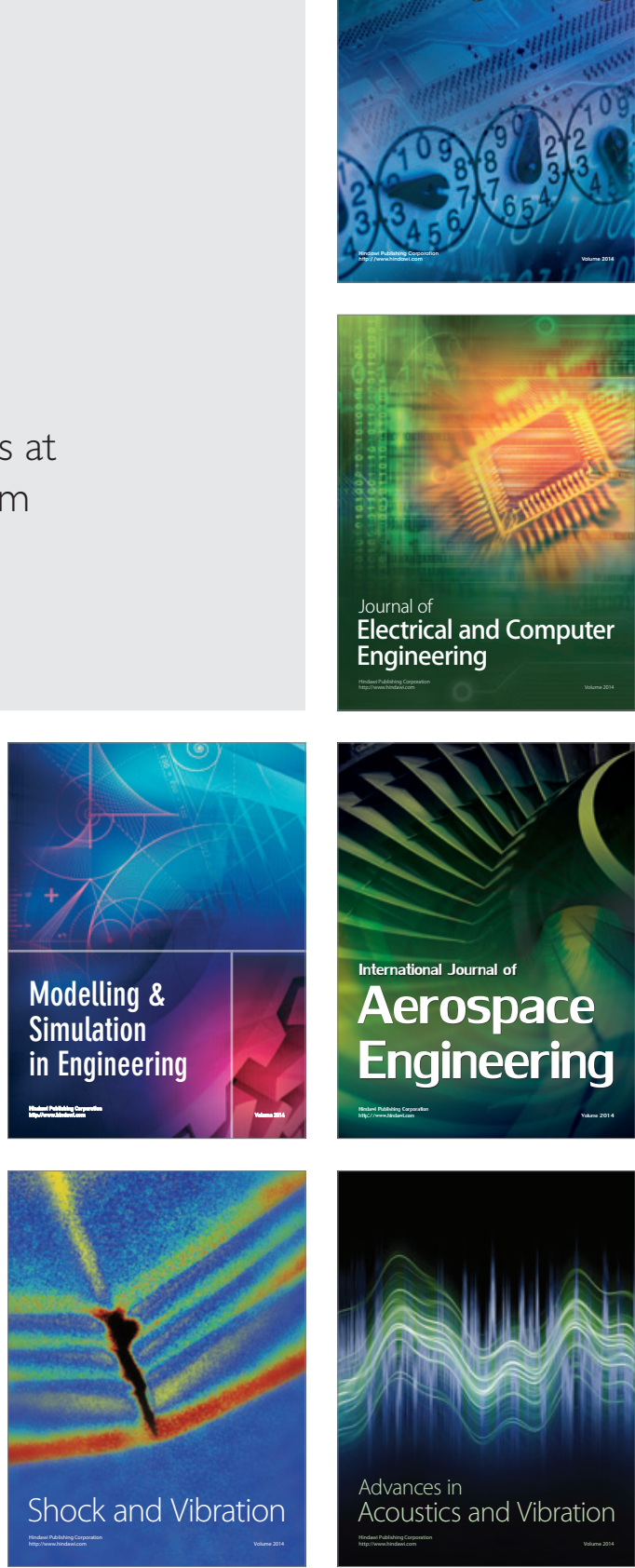